\title{
Implementación de taller de monitores de sutura en alumnos de pregrado de medicina
}

\author{
Roberto González L. 1,2, Héctor Molina Z. ${ }^{2}$, María García-Huidobro D. ${ }^{2,3}$, \\ Patricio Stevens M. ${ }^{2,3}$, Andrés Jadue T. ${ }^{1}$, Alejandra Riquelme U. ${ }^{2}$, \\ Javier Torres M. ${ }^{2}$, Sebastián Barra M. ${ }^{4}$, Felipe Alarcón O. ${ }^{4}$ y Eduardo Fasce H. ${ }^{5}$
}

${ }^{1}$ Centro Cardiovascular, Hospital Clínico Regional de Concepción Dr. Guillermo Grant Benavente. Concepción Chile.

²Departamento de Cirugía Facultad de Medicina, Universidad de Concepción. Concepción, Chile. Servicio de Cirugía Hospital Clínico Regional de Concepción Dr. Guillermo Grant Benavente. Concepción, Chile.

${ }^{4}$ Estudiante Medicina, Facultad Medicina, Universidad de Concepción. Concepción, Chile.

${ }^{5}$ Departamento de Educación Médica, Facultad de Medicina Universidad de Concepción. Concepción, Chile.

Este trabajo se enmarca dentro de la Tesis

titulada "Competencias procedimentales quirúrgicas

básicas en estudiantes

de medicina, enseñanz

práctica por docentes y por

pares", para optar al grado

de Magíster en Educación

Médica para Ciencias de la Salud, a cargo del investigador principal.

Recibido el 2 de julio de 2018 y aceptado para publicación el 17 de julio de 2018

Correspondencia a: Roberto González L. rgonzalezlagos@udec.c
Implementation of suture monitor workshop in undergraduate medical students

Introduction: Suture techniques are considered the minimum of a medical examination and are fundamental in the practice of medicine. Currently, the students of the Universidad de Concepción do not have a formal instance to acquire these competences. Aim: To evaluate the validity of a training program for Suture Instructor (SI) students through the results obtained through a standardized assessment method, carried out in undergraduate students of medicine at the University of Concepción. Method: Preexperimental before and after study. A theoretical-practical intervention was carried out for six medical students of different semesters of the career, between the months of May and July 2017. It consisted of three modules: theoretical classes, practice in biological model and module in surgical pavilion supervised by subspecialists. Continuous and interrupted sutures were evaluated in the biological model using the "The Objective Structured Assessment of Technical Skills" (OSATS) scale. Results of the OSATS scale are described and compared before and after the course. Informed consent was applied. SPSS $®$ was used for statistical analysis by Mann-Whitney test for non-parametric variables. It was considered significant $p<0.05$. Results: All participants improved score on OSATS scale. The participants progressed significantly their results in the different OSATS scales $(5.2 v s 8.8, \mathrm{p}<0.05$ and $16.5 v s 27.2, \mathrm{p}<0.05)$ when comparing pre vs post intervention results respectively. Discussion: Our experience shows that it is possible to create an SI training workshop with excellent results for medical students, through a three phase training program, with theory classes, simulation and "in vivo" practice. It also constitutes the basis of future research that seeks to solve the lack of a formal program of teaching suture techniques to medical students of our university.

Key words: surgery; simulation training; medical education.

\section{Resumen}

Introducción: Las técnicas de sutura básicas se consideran competencias mínimas de un egresado de medicina y son fundamentales en el ejercicio de la medicina general. Actualmente, los estudiantes de la Universidad de Concepción no poseen una instancia formal para adquirir estas competencias. Objetivos: Evaluar la validez de un programa de formación de alumnos monitores de sutura a través de los resultados obtenidos mediante un método de evaluación estandarizado, realizado en estudiantes de pregrado de medicina de la Universidad de Concepción. Metodología: Estudio preexperimental antes y después. Se realizó intervención teórico-práctica para seis estudiantes de medicina de distintos semestres cursados, entre los meses de mayo y julio de 2017. Consistió en tres módulos: clases teóricas, práctica en modelo biológico y módulo en pabellón quirúrgico supervisado por subespecialistas. Se evaluó punto de sutura continuo y discontinuo en modelo biológico mediante la escala "The Objective Structured Assessment Of Technical Skills" (OSATS). Se describen y comparan resultados de la escala OSATS antes y después del curso. Se aplicó consentimiento informado. Se utilizó SPSS ${ }^{\circledR}$ para análisis estadístico mediante prueba de Mann-Whitney para variables no paramétricas. Se consideró significativo $p<0,05$. Resultados: Todos los participantes mejoraron puntuación en escala OSATS. Los participantes progresaron significativamente sus resultados en las distintas escalas OSATS $(5,2 v s 8,8 ; \mathrm{p}<0,05$ y 16,5 vs 27,$2 ; \mathrm{p}<0,05)$ al comparar resul- 
tados pre $v s$ posintervención respectivamente. Discusión: Nuestra experiencia demuestra que es posible crear un taller de formación de MS con muy buenos resultados, para estudiantes de medicina, a través de una capacitación trimodal, con clases teóricas, simulación y práctica in vivo. Además, constituye la base de futuras investigaciones que buscan solucionar la inexistencia de un programa formal de enseñanza de técnicas de sutura a los estudiantes de medicina de nuestra facultad.

Palabras clave: cirugía; entrenamiento de simulación; educación médica.

\section{Introducción}

La sutura de heridas es una técnica fundamental dentro de las competencias quirúrgicas básicas, la primera habilidad quirúrgica que los estudiantes de medicina desean aprender y una de las principales situaciones a la cual se ven enfrentados en el servicio de urgencia de cirugía ${ }^{1}$. Por lo anterior, existe una necesidad evidente de entregar técnicas procedimentales quirúrgicas básicas a estos alumnos, las cuales se consideran competencias mínimas del perfil del egresado y son fundamentales en el ejercicio de la medicina general ${ }^{2}$.

Tradicionalmente las técnicas procedimentales quirúrgicas básicas como suturas, drenajes y aseo quirúrgico de heridas son técnicas que se enseñan tutorialmente en las distintas escuelas de medicina, bajo un ambiente clínico con supervisión docente o de médicos asistenciales. Sin embargo, como toda actividad clínica supervisada, esta experiencia no siempre resulta uniforme para todos los estudiantes debido a diversos factores como disponibilidad horaria, acceso a instrumental especializado, carencia de espacio físico apropiado y características personales de los involucrados ${ }^{3,4}$.

Debido a la falta de estandarización en la enseñanza de técnicas de sutura y bajo la premisa de que las distintas facultades de medicina deben asegurar que estas competencias procedimentales sean adquiridas por sus estudiantes, se han diseñado estrategias de enseñanza-aprendizaje distintas a la tutoría clínica ${ }^{5-9}$. De ellas, el entrenamiento simulado ha sido descrito y validado tanto nacional como internacionalmente en la enseñanza de la cirugía de posgrado $^{10-12}$, fundamentalmente cirugía abierta y laparoscópica, pero existe poca literatura respecto a su implementación en alumnos de pregrado de medicina.

El objetivo de esta comunicación es evaluar la validez de un programa de formación de alumnos Monitores de Sutura (MS) a través de los resultados obtenidos mediante un método de evaluación estandarizado, realizado en estudiantes de pregrado de medicina de la Universidad de Concepción.

\section{Material y Método}

Estudio preexperimental antes y después. Se realizó una intervención teórico-práctica para seis estudiantes de medicina de la Universidad de Concepción entre los meses de mayo y julio de 2017. La intervención consistió en tres módulos: clases teóricas, simulación en modelo biológico y práctica en pabellón quirúrgico, con una duración de 28 horas académicas.

\section{Selección de participantes}

Se convocó a dos estudiantes de sexto, décimo y décimo cuarto semestre de la carrera de medicina según muestreo por conveniencia considerando antecedentes académicos y experiencias de trabajo previas con el equipo investigador. Se solicitó consentimiento informado a los seis participantes y se resguardaron las condiciones éticas correspondientes a la integridad física y mental de los mismos. Se consideró apropiada una muestra de seis participantes fundamentalmente por los recursos disponibles, costos asociados y la implementación de tutoría en pequeños grupos.

\section{Módulo: clases teóricas}

Dos cirujanos plásticos docentes del Departamento de Cirugía dictaron ocho horas académicas sobre tópicos relacionados a técnicas quirúrgicas y suturas, abarcando desde su aparición en la historia y evolución en el tiempo hasta sus indicaciones clínicas actuales. Fue requisito de aprobación la asistencia al $100 \%$ de las clases teóricas y obtener calificación mayor o igual a 6,0 en escala de 1 a 7 en la evaluación de conocimientos al finalizar el módulo.

\section{Módulo: simulación en modelo biológico}

Se utilizó como modelo biológico una pata de cerdo (cruda, refrigerada y no congelada) fijada sobre una tabla de madera a la cual se le realizó una incisión en línea recta de aproximadamente $4 \mathrm{~cm}$ de longitud (Figura 1A). Al comenzar este módulo y sólo con los conocimientos teóricos aprendidos en 


\section{ARTÍ́CULO ORIGINAL}

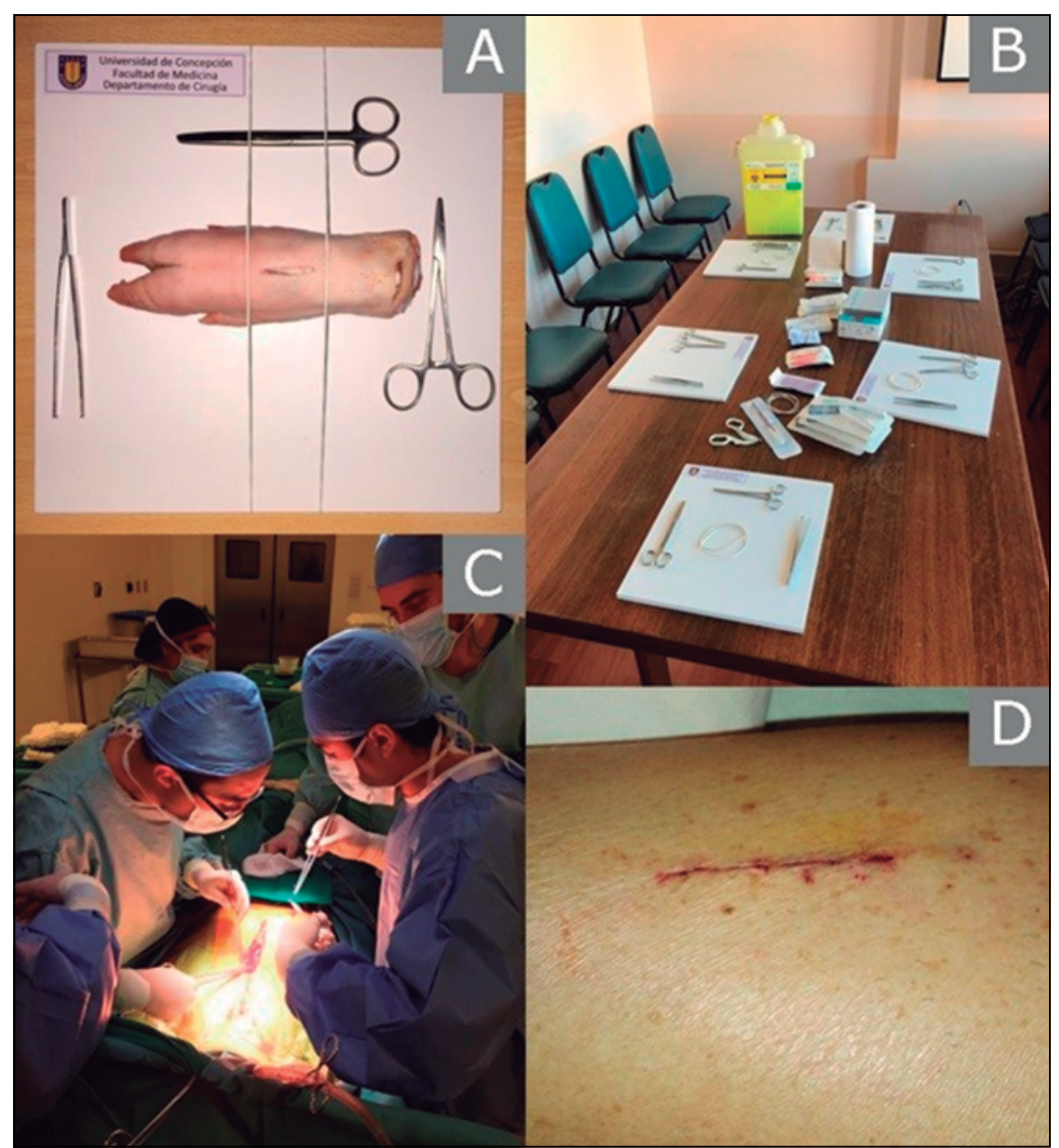

Figura 1. A: Modelo biológico sobre tabla de madera e instrumental quirúrgico utilizado. B: Dependencias del Departamento de Cirugía donde se llevó a cabo la intervención. C: Práctica en pabellón de cirugía cardiotorácica. D: Seguimiento posoperatorio de herida quirúrgica, sin signos de morbilidad. cabo en dependencias del Departamento de Cirugía (Figura 1B).

Adicionalmente, tanto en el módulo de clases teóricas como simulación en modelo biológico, los docentes entregaron herramientas prácticas de docencia y enseñanza en pequeños grupos.

\section{Módulo: práctica en pabellón quirúrgico}

Los participantes cursaron diez horas de intervención en cirugía cardiotorácica donde fueron instruidos in vivo, bajo estricta supervisión de subespecialistas en sutura de distintos planos, en herida quirúrgica de safenectomía y esternotomía (Figura 1C). Se obtuvo consentimiento informado de todos los pacientes previo a la intervención quirúrgica. Se realizó control de la sutura tanto en posoperatorio como seguimiento alejado en policlínico de la especialidad (Figura 1D). Los objetivos de este módulo fueron: integrar los conocimientos de las secciones anteriores, capacitar a los MS en sutura de tejidos vivos bajo condiciones controladas, desarrollar habilidades de campo quirúrgico y técnica aséptica, fortalecer el trabajo en equipo, conocer el ambiente quirúrgico general, además, de realizar seguimiento clínico de la herida operatoria.

Finalizados los tres módulos se realizó la evaluación final que consistió en un ejercicio de punto continuo y discontinuo en modelo biológico con idénticas condiciones al realizado previamente en el módulo de simulación. Nuevamente, los procedimientos fueron registrados en video. Se aplicó una encuesta de satisfacción validada por expertos del Departamento de Educación Médica de la Universidad de Concepción, al finalizar la intervención.

Se consideró como evaluación "pretaller" al registro en video realizado en primera instancia sólo con conocimientos teóricos, previo al inicio del módulo de simulación y como evaluación "postaller" al registro en video realizado al finalizar la totalidad de los módulos. Todas las grabaciones se realizaron con el teléfono móvil personal de cada participante y se hizo entrega de éstos al investigador principal en formato digital.

Para objetivar la progresión de la técnica quirúrgica aprendida se utilizó la escala "Objective Structured Assessment of Technical Skills" (OSATS) ${ }^{13}$ la cual consta de dos secciones: Lista de Comprobación OSATS y Escala Global OSATS. La primera, con un puntaje máximo de 10 puntos, evalúa aspectos procedimentales básicos de forma afirmativa o negativa. Por otro lado, la Escala Global OSATS, con un máximo de 30 puntos, evalúa en escala tipo Likert las habilidades quirúrgicas con énfasis en la coherencia global del procedimiento, otorgando 
Tabla 1. Resultados globales de evaluación pre y postaller

\begin{tabular}{|c|c|c|c|c|c|c|}
\hline & \multicolumn{3}{|c|}{ Lista de comprobación OSATS } & \multicolumn{3}{|c|}{ Escala global OSATS } \\
\hline & Pre taller & Postaller & Progresión absoluta & Pretaller & Postaller & Progresión absoluta \\
\hline$M S 1$ & 6,5 & 9,5 & 3,0 & 19,5 & 25,0 & 5,5 \\
\hline$M S 2$ & 6,5 & 9,0 & 2,5 & 20,5 & 26,0 & 5,5 \\
\hline$M S 3$ & 7,0 & 9,0 & 2,0 & 20,5 & 29,5 & 9,0 \\
\hline$M S 4$ & 3,5 & 7,0 & 3,5 & 10,3 & 26,0 & 15,8 \\
\hline MS 5 & 6,5 & 9,0 & 2,5 & 22,0 & 28,0 & 6,0 \\
\hline$M S 6$ & 1,5 & 9,0 & 7,5 & 6,0 & 28,5 & 22,5 \\
\hline Promedio & 5,3 & 8,8 & 3,5 & 16,5 & 27,2 & 10,7 \\
\hline
\end{tabular}

OSATS: Objective Structured Assessment of Technical Skills, MS: Monitor de Sutura.

un puntaje de 1 si éste resulta ineficiente al 5 si se demuestra un dominio óptimo.

Los videos fueron evaluados por dos cirujanos especialistas que no participaron en la formación de monitores y fueron ciegos tanto al participante como al momento de la grabación.

Los datos se analizaron en el programa SPSS $®$ versión 24. Se describen los resultados pre y posintervención, detallado por variables evaluadas en cada escala OSATS y los resultados de la encuesta de satisfacción. Para analizar el progreso pre versus postaller se utilizó prueba de Mann-Whitney para variables no paramétricas, considerando significativo un valor $\mathrm{p}<0,05$.

\section{Resultados}

Los seis participantes cumplieron con los requisitos de asistencia, evaluación y horas en pabellón quirúrgico, considerados obligatorios para la aprobación del taller.

Los participantes mejoraron su puntuación individual tanto para la Lista de Comprobación OSATS como para la Escala Global OSATS (Tabla 1).

Al analizar a los participantes en conjunto, para la Lista de Comprobación OSATS, se observa que en nueve de las diez variables evaluadas se progresó en términos absolutos al comparar resultados pre versus postaller. La variable "selección de instrumentos" obtuvo el puntaje máximo en ambos momentos de evaluación mientras que "pasa el tejido al primer intento $>80 \%$ " y "tensión correcta de la sutura" fueron las que mostraron mayor y menor progresión, respectivamente (Figura 2).

En la Escala Global OSATS las seis variables evaluadas presentaron progreso de puntuación al

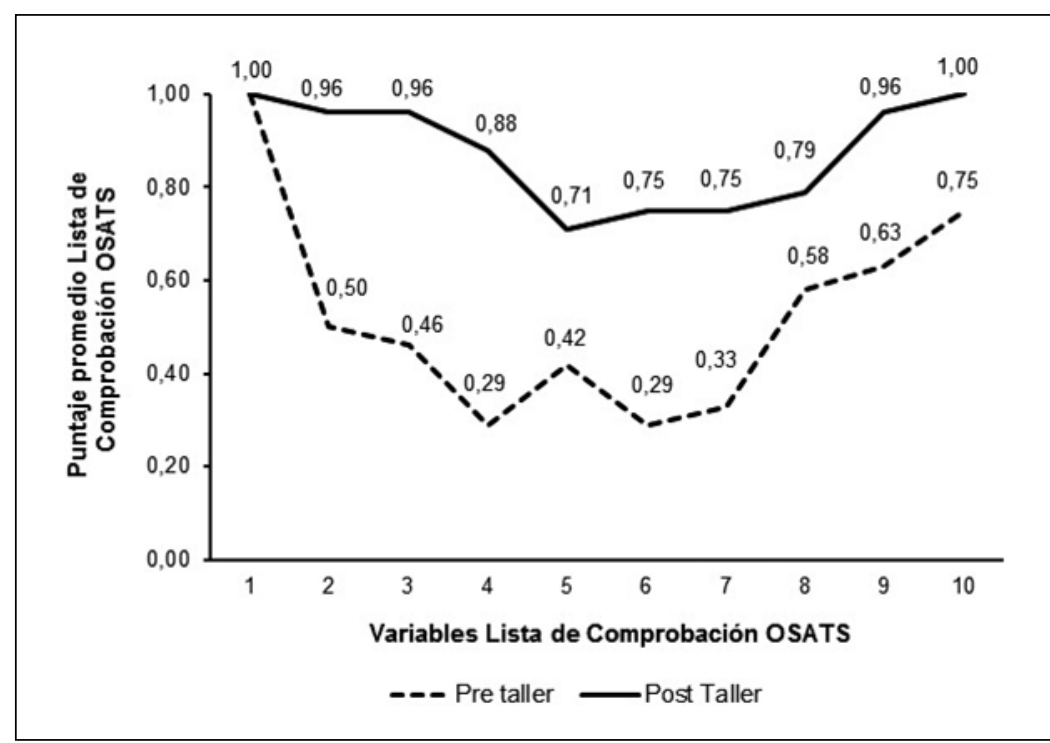

Figura 2. Detalle de resultados según variable evaluada en Lista de Comprobación OSATS Variables Lista de Comprobación OSATS: (1) Selección de instrumentos, (2) Utiliza la sutura adecuada, (3) Ubica la sutura correctamente en el porta y la introduce adecuadamente en el tejido, (4) Pasa el tejido al primer intento (> 80\%), (5) Recupera la aguja del tejido correctamente (>90\%), (6) No daña los tejidos con las pinzas, (7) Hay una adecuada separación de los puntos, (8) Tensión correcta en la sutura, (9) No deja restos de sutura en el campo quirúrgico, (10) No pierde la aguja.

comparar resultados pre versus postaller. Las variables "planificación y ritmo" y "conocimiento del procedimiento específico" fueron las mejor evaluadas con un promedio de 4,8 puntos para un máximo de 5,0. "Uso apropiado del instrumental" mostró la menor progresión dentro de esta escala (Figura 3).

El resultado global de la intervención usando la Lista de Comprobación OSATS, evidencia un aumento del promedio pre taller desde 5,3 $\pm 1,3$ hasta $8,8 \pm 0,7$ postaller. Este aumento es estadísti- 


\section{ARTÍ́CULO ORIGINAL}

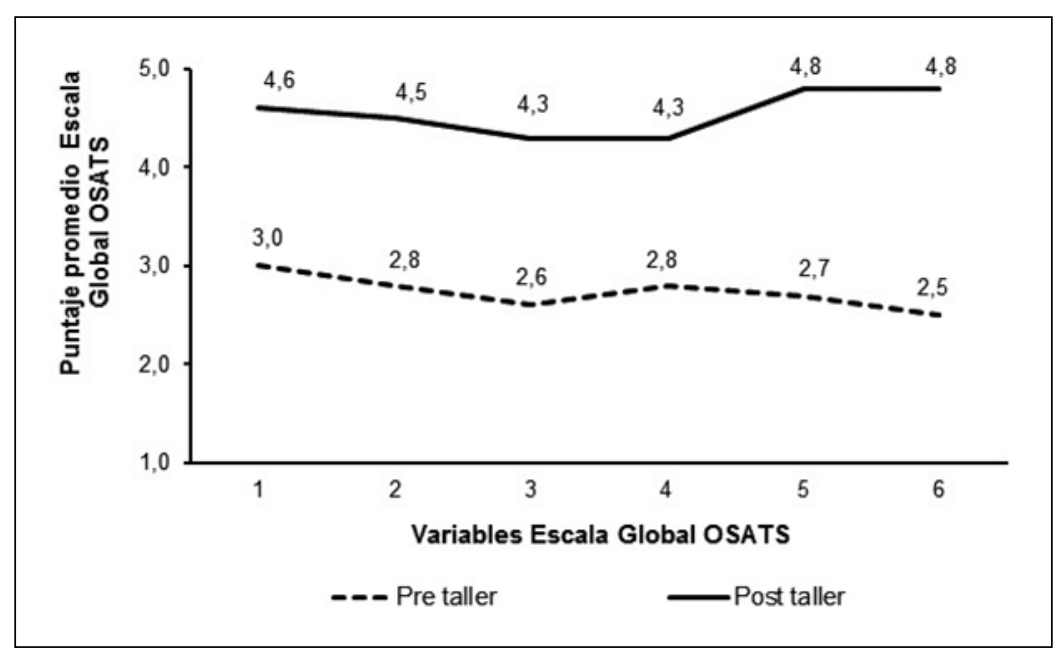

Figura 3. Detalle de resultados según variable evaluada en Escala Global OSATS. Variables Escala Global OSATS: (1) Manejo de tejidos, (2) Tiempo y motricidad, (3) Manipulación de instrumentos, (4) Uso apropiado de instrumental, (5) Planificación, (6) Conocimiento del procedimiento. zar el taller, los participantes calificaron con máxima puntuación los distintos ítems que evaluaban calidad docente, suficiencia de horas en práctica e idoneidad del modelo biológico como prototipo de simulación. El taller en general fue calificado con nota 7,0.

Se realizó control de la herida quirúrgica durante el posoperatorio y seguimiento alejado en policlínico de la especialidad, sin encontrar morbilidad en los pacientes.

\section{Discusión}

La falta de estandarización en la enseñanza de técnicas de sutura provoca que muchas, veces los estudiantes de medicina, adquieran estas competencias durante su internado cuando se ven obligados a suturar a pacientes, dependiendo no solamente de la exposición a una situación de urgencia, sino también de la voluntad de los médicos asistenciales y/o becarios de cirugía.

Esto repercute directamente en la autopercepción de los médicos recién egresados al consultarles por la adquisición de competencias durante la carrera de medicina, ya que se declaran como poco competentes cuando se evalúan competencias procedimentales quirúrgicas versus competencias médicas ${ }^{14}$.

El advenimiento de la simulación clínica como modelo estandarizado para el entrenamiento en

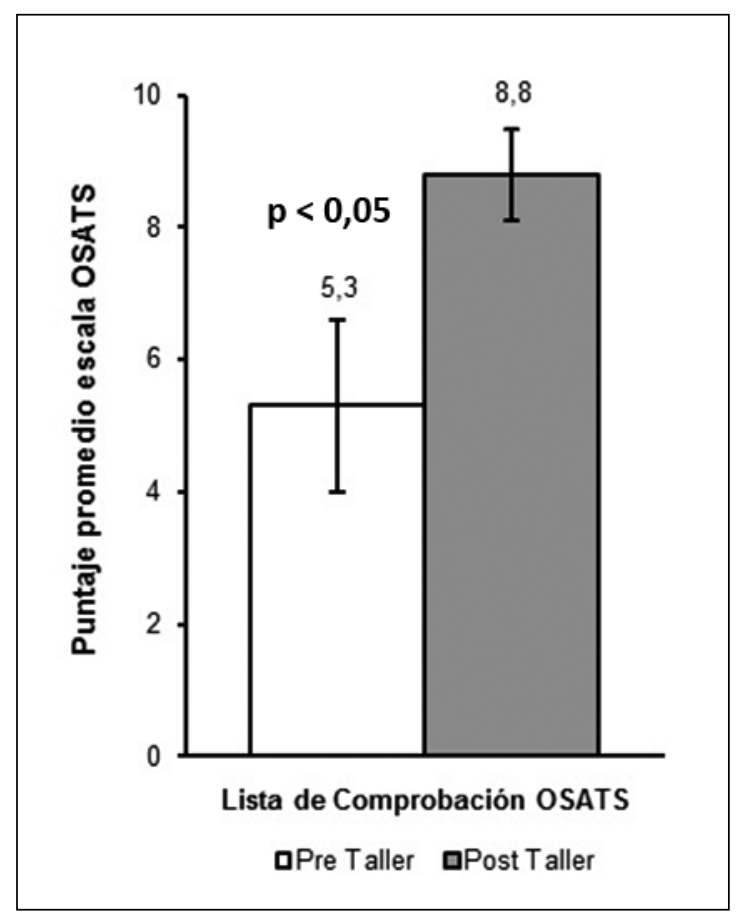

Figura 4. Resultados Lista de Comprobación OSATS.

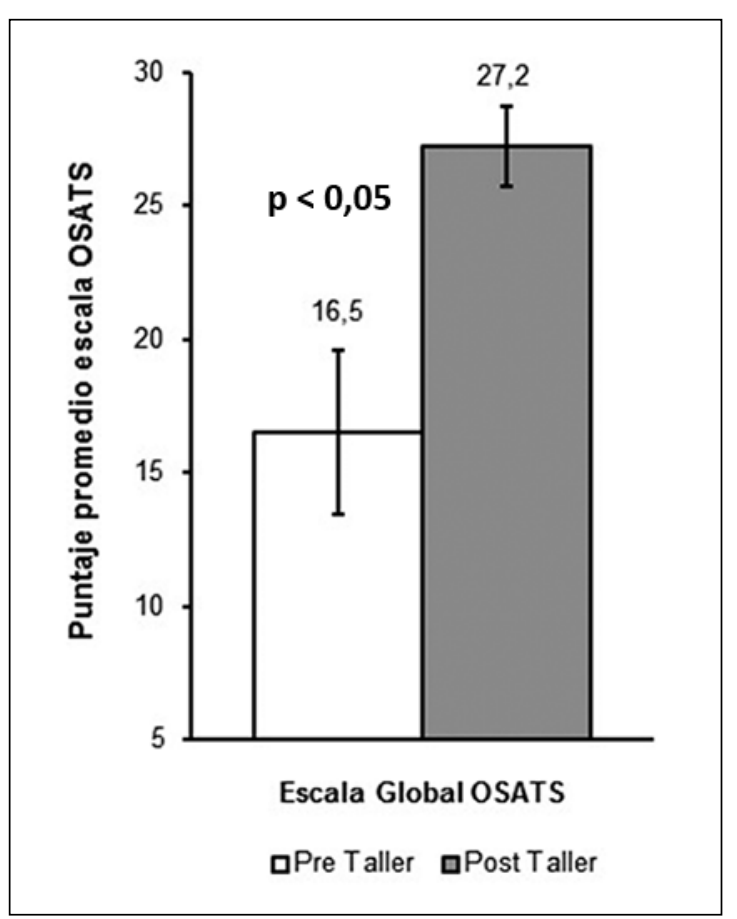

Figura 5. Resultado Escala Global OSATS. 
competencias quirúrgicas ha demostrado optimizar recursos, disminuir tiempos de práctica y acortar curvas de aprendizaje. Además, al utilizar pautas de evaluación estandarizadas se disminuye el sesgo del modelo tutorial operador-dependiente, siendo bien acogido tanto por docentes como estudiantes ${ }^{15}$.

Nuestra experiencia demuestra que es posible implementar un taller de formación de MS para estudiantes de medicina, a través de una capacitación dirigida en aspectos teóricos básicos, de simulación en modelo biológico y práctica in vivo en pabellón quirúrgico.

Además, se obtuvo buenos resultados usando una pata de cerdo como modelo de simulación, principalmente por su bajo costo y ventajas de manipulación. Si bien este modelo presenta diferencias obvias con la piel humana, la práctica in vivo pretende salvaguardar el objetivo intrínseco, que es formar monitores competentes en las técnicas quirúrgicas básicas.

Los participantes mejoraron sus puntuaciones en las distintas escalas OSATS con progresos estadísticamente significativos. No está claro si existe una relación proporcional entre la cantidad de horas de entrenamiento y la progresión de las habilidades quirúrgicas aprendidas, sin embargo, estudios nacionales han demostrado resultados similares con intervenciones teórico-prácticas en distintos modelos de simulación, manteniendo adecuada validez de contenido al evaluar los procedimientos con escalas OSATS $^{16}$.

Si bien este estudio no permite evaluar el impacto aislado que tiene la práctica en pabellón quirúrgico in vivo en la adquisición de habilidades quirúrgicas básicas, sí permitió realizar seguimiento posoperatorio y alejado de la herida quirúrgica evidenciando ausencia de morbilidad y muy buenos resultados, aunque no estandarizados. Al mismo tiempo se logró la adquisición de otras competencias anexas a la técnica de sutura como relación con el ambiente quirúrgico, implementación de campo estéril y trabajo en equipo.

La principal limitante para la replicación de este estudio radica en la disponibilidad de los docentes que la imparten, lo que hace particularmente difícil repetir esta experiencia en casas de estudios que no tengan un amplio recurso de cirujanos o pabellones quirúrgicos dispuestos para este fin.

La dificultad de contar con un número de docentes cirujanos, suficiente para la enseñanza personalizada de técnicas de sutura básicas en pequeños grupos ha motivado la utilización de alumnos ayudantes para la enseñanza de dichas competencias. Por lo anterior, esta comunicación contribuye con una metodología para la formación de alumnos MS, procurando que posean mayor experiencia y horas de entrenamiento en técnicas quirúrgicas básicas, además, de una adecuada preparación por parte de docentes del mismo Departamento de Cirugía, con la intención de implementar una intervención a futuro en la cual estos monitores puedan entregar sus conocimientos aprendidos mediante enseñanza práctica por pares, a otros estudiantes de medicina, aplicada en modelo biológico.

Actualmente, nuestro grupo trabaja en la implementación de un taller de suturas para todos los alumnos de la facultad, bajo tutoría de MS y será motivo de nuevas investigaciones lo que nos permitirá evaluar el real impacto de este taller de formación.

En suma, este taller constituye un avance en la búsqueda de un programa formal de enseñanza de técnicas de sutura, para estudiantes de medicina de la Universidad de Concepción.

\section{Responsabilidades éticas}

Protección de personas y animales. Los autores declaran que los procedimientos seguidos se conformaron a las normas éticas del comité de experimentación humana responsable y de acuerdo con la Asociación Médica Mundial y la Declaración de Helsinki.

Confidencialidad de los datos. Los autores declaran que han seguido los protocolos de su centro de trabajo sobre la publicación de datos de pacientes.

Derecho a la privacidad y consentimiento informado. Los autores declaran que en este artículo no aparecen datos de pacientes.

\section{Referencias}

1. Huang E, Vaughn C, Chern H, O'Sullivan P, Kim E. An objective assessment tool for basic surgical knot-tying skills. J Surg Educ. 2015;72:572-6.
2. Cumming A, Ross M. The Tuning Project for Medicine-learning outcomes for undergraduate medical education in Europe. Med Teach. 2007;29:636-41.

3. Zundel S, Meder A, Ziofel S, HerrmannWerner A. The surgical experience of current non-surgeons gained at medical school: a survey analysis with implications for teaching today's students. BMC Med Educ. 2015;15:187.

4. Colliva de Boada, M. Taller de cierre de heridas una estrategia metodológica 


\section{ARTÍ́CULO ORIGINAL}

de apoyo a la enseñanza práctica de la cirugía. Revista Salus [revista en internet] 1999; 3: Disponible en: http://servicio. bc.uc.edu.ve/fcs/vol3n2/5taller.pdf

5. Jiménez M, Hernández J, Martínez F, Rodríguez, $\mathrm{M}^{\mathrm{a}} \mathrm{J}$. Avances en metodología de evaluación de estudiantes en Habilidades Clínicas y Quirúrgicas Básicas (HCQB) en Medicina. En: EVALfor Ed, EVALtrends 2011 -

Evaluar para aprender en la universidad: Experiencias innovadoras en la sistematización de la evaluación; 2011. P. 291-303

6. Torres R, Orban R, Serra E, Marecos, Vargas L, Deffis L, et al. Enseñanza de técnicas quirúrgicas básicas en simuladores biológicos. Experiencia pedagógica en el pregrado. Educación Médica 2003;6:149-52.

7. Montesinos M. Enseñanza de cirugía basada en competencias en el pregrado de medicina. Rev Argent Cirug. 2013;104:7785.

8. Nousiainen M, Brydges R, Backstein D, Dubrowski A. Comparison of expert instruction and computer-based video training in teaching fundamental surgical skills to medical students. Surgery 2008;143:539-44.

9. Quirarte C, Muñoz J. La revolución pedagógica en la cirugía, parte I. Los agentes del cambio. Cirugía Endoscópica 2013;14:7-22.

10. Martin J, Regehr G, Reznick R, MacRae $\mathrm{H}$, Hutchison C, Brown M. Objective structured assessment of technical skills (OSATS) for surgical residents. Br J Surg. 1997;84:273-8.

11. Castillo R, Buckel E, León F, Varas J, Alvarado J, Achurra P, et al. Effectiveness of learning advanced laparoscopic skills in a brief intensive laparoscopy training program. J Surg Educ. 2015;72:648-53.
12. Arribalzaga E, Javovella P. Estudio observacional de habilidades quirúrgicas en residentes. Educación Médica 2006;9:27-34.

13. Moorthy K, Munz Y, Sarker S, Darzi A. Objective assessment of technical skills in surgery. BMJ 2013;327:1032-7.

14. Villegas F, Polaco A, González J, García A, Madrid Ml. Competencias médicoquirúrgicas. Autopercepción en médicos recién egresados de la licenciatura. Cir Ciruj. 2007;75:43-7.

15. Graafland M, Schraagen J, Schijven M. Systematic review of serious games for medical education and surgical skills training. Br J Surg. 2012;99:1322-30.

16. Alvarado J, Henríquez J, Castillo R, Sosa J, León F, Varas J, et al. Programa pionero de simulación en sutura para estudiantes de medicina de pregrado. Rev Chil Cir. 2015;67: 480-5. 